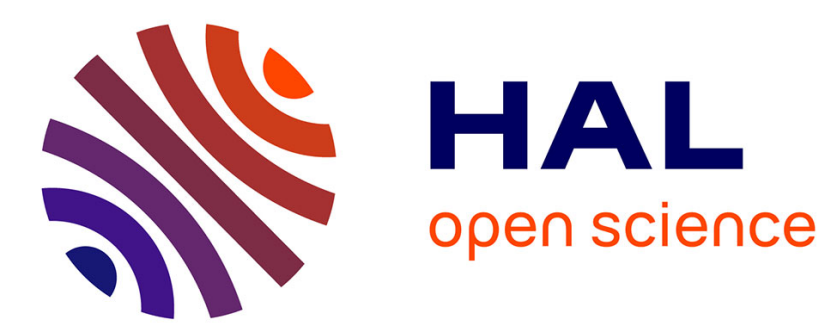

\title{
INTERACTION ENTRE UN CHAMP SONORE STATIONNAIRE INTENSE ET UN ÉCOULEMENT TURBULENT GUIDÉ
}

\author{
Laurent-Charles Valdes
}

\section{- To cite this version:}

Laurent-Charles Valdes. INTERACTION ENTRE UN CHAMP SONORE STATIONNAIRE INTENSE ET UN ÉCOULEMENT TURBULENT GUIDÉ. Journal de Physique Colloques, 1990, 51 (C2), pp.C2-1205-C2-1208. 10.1051/jphyscol:19902283 。 jpa-00230616

\section{HAL Id: jpa-00230616 https://hal.science/jpa-00230616}

Submitted on 1 Jan 1990

HAL is a multi-disciplinary open access archive for the deposit and dissemination of scientific research documents, whether they are published or not. The documents may come from teaching and research institutions in France or abroad, or from public or private research centers.
L'archive ouverte pluridisciplinaire HAL, est destinée au dépôt et à la diffusion de documents scientifiques de niveau recherche, publiés ou non, émanant des établissements d'enseignement et de recherche français ou étrangers, des laboratoires publics ou privés. 
COLLOQUE DE PHYSIQUE

Colloque C2, supplément au $\mathrm{n}^{\circ} 2$, Tome 51, Février 1990

ler Congrès Français d'Acoustique 1990

INTERACTION ENTRE UN CHAMP SONORE STATIONNAIRE INTENSE ET UN ECOULEMENT TURBULENT GUIDE

L. - C, VALDÈS

Office National d'Etudes et de Recherches Aérospatiales, BP. 72, F-92322 Châtilion Cedex, France

Résumé - Une interaction peut résulter de la présence d'un système d'ondes planes stationnaires dans un écoulement turbulent guidé dans un tube. Elle se traduit par une forte diminution de la perte de charge singulière d'entrée et n'a lieu que si les vitesses acoustique et aérodynamique ont des composantes perpendiculaires entre elles et si le champ sonore est stationnaire.

Abstract - Interaction between a high-level steady acoustic field and a ducted turbulent flow. An interaction can arise from a steady standing wave in a ducted turbulent flow. It induces a strong decrease of pressure loss. It only appears if acoustic and aerodynamic velocities have perpendicular components, and if the acoustic field is steady.

\section{1 - INTRODUCTION}

La découverte du phénomène d'excitation des jets libres par une onde sonore [1] a dégagé un point de vue nouveau où 1 'acoustique est envisagée comme un moyen d'agir sur l'aérodynamique $[2,3,4]$. Les expériences qui font 1 'objet de cette communication sont conçues pour élucider certains mécanismes $d^{\prime}$ interaction entre un champ sonore et un écoulement turbulent.

\section{2 - LE DISPOSITIF EXPERIMENTAL}

Le montage expérimental (fig. 1) comprend un tube de $6 \mathrm{~m}$ de longueur et de $50 \mathrm{~mm}$ de diamètre intérieur, parcouru par un écoulement d'air dont la vitesse moyenne $U$ se situe entre lo et $30 \mathrm{~m} / \mathrm{s}$. Un système d'ondes planes stationnaires ou quasi-stationnaires est produit par deux sources sonores placées en vis-à-vis aux extrémités du tube. Le niveau sonore est compris entre 130 et $150 \mathrm{~dB}$, de sorte que 1 'énergie cinétique acoustique soit du même ordre de grandeur que 1 'énergie cinétique des fluctuations turbulentes.

Des tubes de Pitot, associés à des prises de pression statique, équipent les sections (1) et (2). Ils permettent la mesure de la perte de charge $\pi$ en amont de (1). et de $\pi^{\prime}$ entre (1) et (2). Cette dernière est assimilée à la perte de charge linéique. Des profils de vitesse sont mesurés dans la section (1).

Les mesures acoustiques consistent en une exploration longitudinale du champ sonore au moyen d'un microphone glissé dans le tube. On en tire la courbe de pression sonore efficace $p_{e f f}(x)$.

\section{3 - EXISTENCE D'UNE INTERACTION}

Une expérience probatoire a d'abord établi l'existence d'une interaction. L'écoulement a une vitesse moyenne $U=30 \mathrm{~m} / \mathrm{s}$. Il entre par des fentes latérales diamétralement opposées. Les fils de laine utilisés pour visualiser les lignes de courant indiquent les changements de rotation rapides et aléatoires. Avec une excitation acoustique de $150 \mathrm{~dB}$ a $900 \mathrm{~Hz}$, les fils s'immobilisent parallèlement à 1 'axe, tandis que $\pi$ diminue notoirement.

Après cette première expérience, il s'agissait, d'une part, de préciser les conditions requises pour observer cette interaction et, d'autre part, de la caractériser quantitativement si possible. 


\section{4 - CONDITIONS REQUISES POUR L'INTERACTION}

Différentes terminaisons peuvent être montées à l'amont du dispositif expérimental. Elles déterminent le type d'écoulement (tourbillonnaire ou axial) et de champ sonore (purement stationnaire ou non selon que deux sources ou une seule sont utilisées). Les expériences sont réalisées dans chacune de ces configurations expérimentales et 1'interaction est évaluée par $\Delta=1-\pi$ (avec acoustique) $/ \pi$ (sans acoustique).

Les résultats obtenus sont synthétisés dans le tableau ci-dessous. Il en ressort que l'interaction a lieu seulement lorsque l'écoulement est tourbillonnaire et que le champ sonore est stationnaire (2 sources). La vitesse acoustique, axiale puisque les ondes sont planes, interagit donc avec la composante de la vitesse aérodynamique qui lui est perpendiculaire. L'interaction pourrait provenir de la composante réactive de l'intensité sonore, qui se rapporte à la part de l'énergie sonore qui ne se propage pas.

\begin{tabular}{|l|c|c|}
\hline & 2 sources sonores & 1 source sonore \\
\hline Ecoulement tourbilionnaire & $\Delta \neq 0$ & $\Delta \simeq 0$ \\
Ecoulement axial & $\Delta=0$ & $\Delta=0$ \\
\hline
\end{tabular}

\section{5 - CARACTERISATION DE L'INTERACTION}

Une exploration en fréquence réalisée à niveau sonore maximal montre que 1 'interaction a 1 ieu d'une façon modérée $(\Delta \simeq 7 \%)$ dans la bande de fréquence comprise entre 100 et $2000 \mathrm{~Hz}$, sauf pour quelques fréquences discrètes où elle est notable $(\Delta \simeq 30 \%)$.

$L^{\prime}$ intensité de $l^{\prime}$ interaction a été étudiée en fonction du niveau acoustique, a la fréquence qui rend maximum 1'écart $\pi$ (sans acoustique) - $\pi$ (avec acoustique). Le paramètre aérodynamique est $\Delta$ et le paramètre acoustique est la pression sonore maximale $p_{\text {max }}$ relevée sur la courbe de $p_{e f f}(x)$. Les résultats (fig. 2) montrent que $\Delta$ croît avec $p_{\text {max }}$ jusqu'à une valeur de saturation de $l^{\prime}$ ordre de $30 \%$ et que $l^{\prime}$ augmentation de $\Delta$ avec $p_{\text {max }}$ est plus importante aux basses vitesses $d^{\prime}$ écoulement $U$.

L'interaction $s^{\prime}$ accompagne $d^{\prime}$ une augmentation de $\pi^{\prime}$ mais celle-ci est toujours marginale devant la diminution de $\pi$, dont $\eta^{\prime}$ essentiel provient de la perte de charge singulière d'entrée. L'interaction concerne donc exclusivement cette dernière. La diminution de $\pi$ $s^{\prime}$ accompagne d'une très nette modification du profil radial de vitesse (fig. 3 ) ; le champ sonore lui donne une allure parabolique, caractéristique des écoulements turbulents pleinement établis.

La croissance de $\Delta$ avec $p_{\text {max }}$ indique que le champ sonore diminue la puissance dissipée par frottements visqueux. Quant à $l^{\prime}$ influence de l'écoulement sur le champ sonore (fig. 4), elle se traduit toujours par la diminution de l'aire sous la courbe $p_{e f f}(x)$, indiquant une augmentation de la puissance sonore absorbée dans 1'écoulement.

\section{6 - CONCLUSION}

L'effet le plus notable de $l^{\prime}$ interaction est certainement la diminution, pouvant atteindre $30 \%$, de 1 a perte de charge singulière d'entrée. Toutefois, cette interaction n'intervient que lorsque les vitesses acoustique et aérodynamique ont des composantes perpendiculaires entre elles et que le champ sonore est stationnaire. Ces expériences soulèvent la question du mécanisme qui permet de réduire la puissance dissipée par frottements visqueux par absorption de puissance sonore, en apportant toutefois certains éléments de réponse.

\section{REMERCIEMENTS}

Le dispositif expérimental a été mis au point par deux élèves ingénieurs de 1'ENSAM, M. Frère et Mlle Loiseau, dans le cadre de leur projet de fin d'étude. Ils ont contribué a dégager les principaux résultats cités dans cette communication.

\section{REFERENCES}

[1] Bechert, D. and Pfizenmaier, E., J. Sound Vib. 43 (1975) 581.

[2] Ffowcs Williams, J.E., Inter-noise 88 Proceedings (1988) 5.

[3] Lowson, M., Experiments on acoustic forcing of three-dimensional shear layers, AIAA-89-1063 (1989).

[4] Shearin, J. and Jones, M., Airfoil profile drag increase due to acoustic excitation, AIAA-89-1069 (1989). 


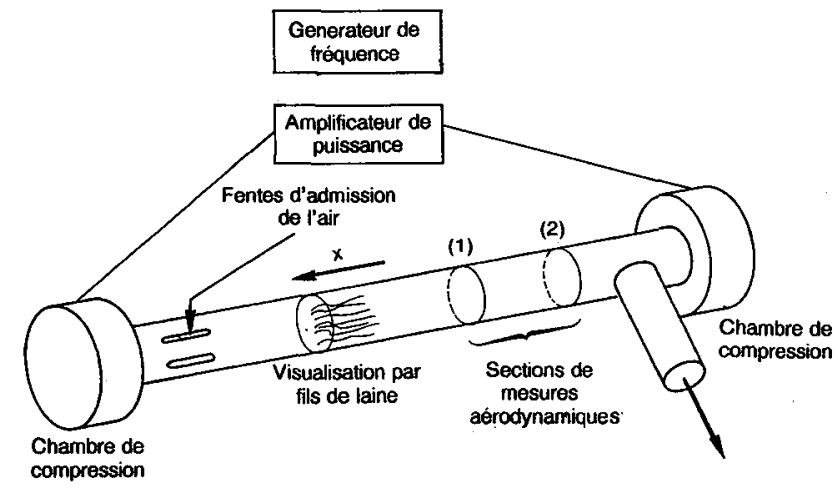

Fig. 1 - Schéma du montage expérimental.

Fig. 2 - Evolution des pertes de charge avec la pression sonore.
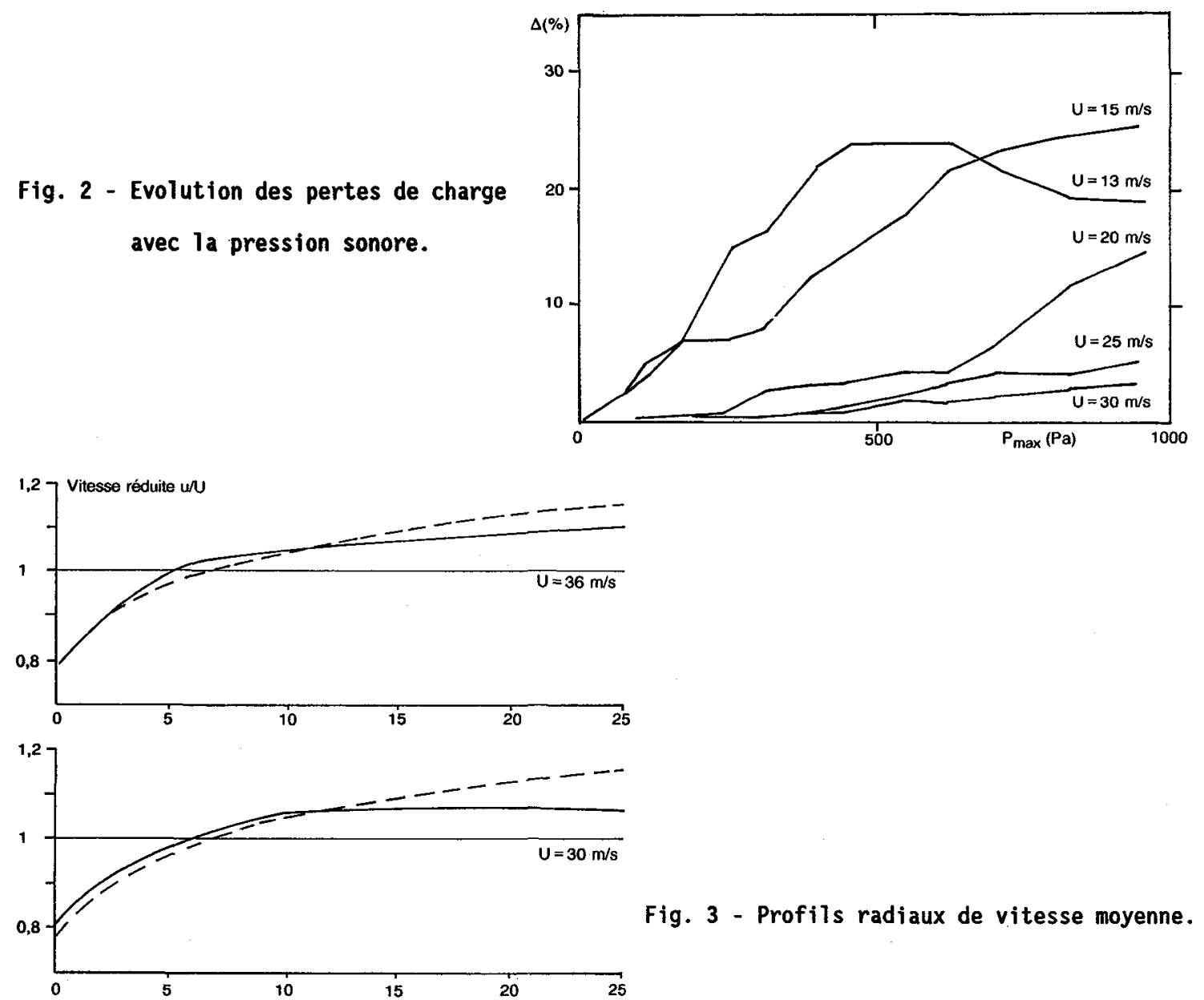

Fig. 3 - Profils radiaux de vitesse moyenne.

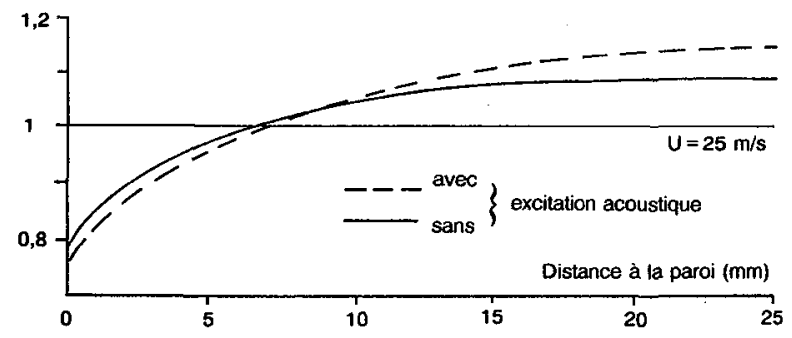




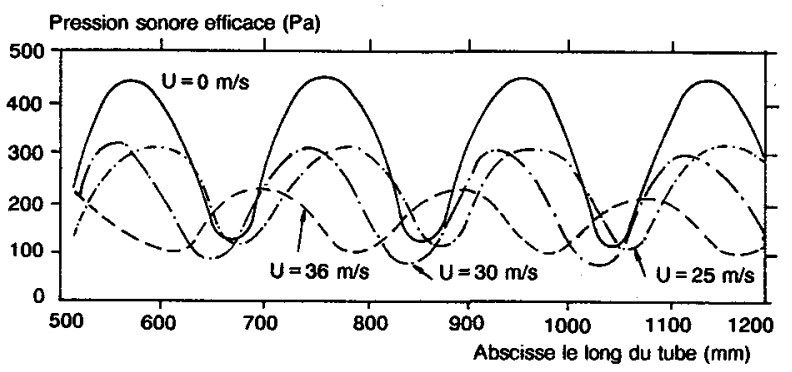

Fig. 4 - Evolution longitudinale de la pression sonore. 